\title{
COMO AS CAPACIDADES DE MARKETING DETERMINAM A ESCOLHA DOS MODOS DE ENTRADA NO ESTRANGEIRO E A SELEÇÃO DOS PAÍSES DE DESTINO
}

\author{
Thiago Chiorino Costa $^{1}$, Manuel Portugal Ferreira ${ }^{\Omega}$, Julio Araujo Carneiro da Cunha, Cláudia Frias Pinto ${ }^{€}$ \\ $\Omega$ Universidade Federal de Lavras - UFLA, Minas Gerais (Brasil) \\ €Universidade do Sul de Santa Catarina - UNOESC, Santa Catarina (Brasil) \\ Universidade Nove de Julho - UNINOVE, São Paulo (Brasil)
}

\section{DETALHES DO ARTIGO}

Recebido: 08 de março de 2019

Aceito: 20 de novembro de 2019

Disponível online: 01 de jan. de 2020

Sistema de revisão "Double blind review"

Editor Científico

Ilan Avrichir

\section{Palavras-chaves:}

Capacidades de marketing

Modos de entrada

Seleção dos mercados

\section{RESUMO}

Objetivo: Neste artigo analisamos, conceitualmente, a influência das capacidades de marketing como antecedentes de duas decisões basilares na internacionalização das empresas: a seleção do modo de entrada no estrangeiro e do país de destino. Método: Utilizamos a tipologia com quatro tipos de capacidades de marketing especializadas, multifuncionais, arquiteturais e dinâmicas - para observar como podem, de modos diferenciados, conduzir a diferentes escolhas de países de destino (localização) e modos de entrada.

Principais resultados: Propomos que capacidades de marketing de nível mais alto induzem a internacionalização para países mais desenvolvidos e usando modos de entrada de maior controle e comprometimento de recursos, e em contraste, capacidades de nível mais baixo a escolha de mercados de desenvolvimento idêntico ou inferior e a modos de entrada de menor controle e comprometimento de recursos

Relevância/originalidade - $O$ nosso trabalho explora uma possível relação das capacidades de marketing detidas pelas empresas e sua influência quanto a escolha do modo de entrada em mercados externos e quanto a seleção do país de destino. Contribuições teóricas: Necessário Complementamos a literatura existente sobre o papel das capacidades na internacionalização das empresas, especificamente observando as capacidades de marketing que se revelam mais próximas do mercado, em contraste com pesquisa sobre competências baseadas em conhecimento tecnológico ou inovação, operacionais ou capacidades de fazer arbitragem entre mercados.

\section{Introdução}

A pesquisa em negócios internacionais tem sido abundante em entender os fatores que determinam quer os modos de entrada no estrangeiro (Andersen, 1997; Hennart \& Slangen, 2015), quer a seleção dos mercados estrangeiros (Johanson \& Vahlne, 1977; Musso \& Francioni, 2014). Estas duas linhas de pesquisa têm explorado vertentes teóricas distintas como a teoria dos custos de transação (Hennart \& Park, 1993; Zhao et al., 2004), Visão Baseada nos Recursos (Anand \& Delios, 2002; Meyer, Wright \& Pruthi, 2009; Cavusgil \& Knight, 2015) e Teoria Institucional (Cuervo-Cazurra \& Genc, 2008; Wu, 2013; Gaur et al., 2014). No entanto, a pesquisa tem sido menos profícua em explorar como as capacidades de marketing detidas pelas empresas podem influenciar a escolha do modo de entrada em mercados externos e a seleção do país de destino. Esta lacuna é talvez especialmente relevante no contexto das empresas de economias emergentes que não detém nem grande experiência internacional de operar nos mercados externos (Cuervo-Cazurra \& Genc, 2008; Cuervo-Cazurra, 2015), nem os recursos financeiros, mas que podem ter desenvolvido internamente capacidades, ou capabilities, e em especial capacidades de marketing, passíveis de serem exploradas nos mercados externos (Blesa \& Rippoles, 2008; Kaleka \& Morgan, 2017; Konwar et al., 2017).

A pesquisa em marketing tem intensificado o foco na conceituação de capacidades de marketing, também explorando empiricamente como as 
capacidades de marketing influenciam o desempenho das empresas (Kamboj \& Rahman, 2015). As capacidades de marketing manifestam-se na habilidade de as empresas usarem os recursos disponíveis para executar tarefas de marketing para alcançar os resultados de marketing desejados (Morgan, Katsikeas \& Vorhies, 2012). A literatura sugere que as capacidades de marketing têm características de recursos estratégicos, sendo valiosas (Dutta et al., 1999), inimitáveis (Morgan et al., 2009) e não substituíveis podendo conceder uma vantagem competitiva sustentável e desempenho superior das empresas (Krasnikov \& Jayachandran, 2008).

Em marketing internacional, Tan e Sousa (2015) mostraram que as capacidades de marketing melhoram o desempenho das empresas (ver, também, Blesa \& Rippoles, 2008), possivelmente, como sugerem Kotabe et al. (2002) moderando a relação entre a multinacionalidade e o desempenho das empresas. Por outro lado, as capacidades de marketing desenvolvidas nos mercados domésticos parecem contribuir para as empresas terem melhor desempenho internacional, mas independentemente do país de origem (Blesa \& Rippoles, 2008). Outras pesquisas relacionadas com as capacidades de marketing sugerem que a intensidade e investimentos em vendas e propaganda podem influenciar a escolha dos modos de entrada (Anand \& Delios, 2002; Chen, 2008), sendo que capacidades de marketing desenvolvidas aumentariam a probabilidade de entradas em outros mercados por meio de aquisições. E há evidências de que as empresas que se internacionalizam para mercados emergentes têm um nível substancialmente superior de capacidades de marketing em comparação com empresas que apenas exportam para mercados desenvolvidos (Bortoluzzi et al., 2014), mas não é evidente que tipo de capacidades de marketing têm o potencial de ter maior valor para operar em mercados menos desenvolvidos em comparação com mercados mais desenvolvidos.

Neste estudo conceitual propomos que as capacidades de marketing, como apresentadas por Morgan (2012) e Morgan et al. (2018), a saber, capacidades especializadas, capacidades arquiteturais, capacidades multifuncionais e as capacidades dinâmicas de marketing, podem influenciar as decisões relativas quer à seleção dos modos de entrada, quer a escolha do país de destino. Sendo as capacidades de marketing relacionadas ao mercado, o melhor entendimento delas pode auxiliar na análise de quais recursos materiais ou imateriais, ou outros tipos de capacidades tecnológicas ou de gestão, podem favorecer as estratégias para a internacionalização das empresas. Estendemos, portanto, conceitualmente a pesquisa existente para analisar como um conjunto de capacidades de marketing são possíveis preditores dos modos de entrada no estrangeiro, e da seleção dos mercados externos onde entrar. O argumento central assenta em que empresas com melhor desempenho em níveis mais baixos de capacidades de marketing (que definiremos como as capacidades de marketing especializadas ou multifuncionais) tendem a preferir modos de entrada no estrangeiro por meio de exportações ou joint-ventures e, em contraste, as empresas que possuem melhor desempenho em níveis mais altos de capacidades de marketing (definidas como as capacidades de marketing arquiteturais ou dinâmicas) tenderão a preferir modos de entrada no estrangeiro por meio de aquisições ou investimentos greenfield (ou de startup).

De forma similar, argumentamos que as empresas que possuem níveis mais baixos de capacidades de marketing tenderão a escolher países menos desenvolvidos do que o seu país de origem, enquanto, em contraste, as empresas que possuem níveis mais altos de capacidades de marketing tenderão a escolher países com maior nível de desenvolvimento que o seu país de origem.

Este estudo tem duas contribuições conceituais. Primeiro, expande sobre a literatura existente reforçando a conceitualização de capacidades de marketing (Vorhies \& Morgan, 2005; Morgan, 2012; Morgan et al., 2018), e comparando e contrastando os efeitos de diferentes capacidades de marketing. Não é, assim, apenas uma questão de saber se as empresas detêm as capacidades, mas quais são as capacidades detidas e qual o seu impacto diferenciado. Segundo, o estudo estende a conceitualização das capacidades de marketing especificamente para o contexto dos negócios internacionais e para duas decisões fulcrais na pesquisa em negócios internacionais - a escolha dos modos de entrada no estrangeiro e das localizações adicionando, assim, ao leque de possíveis capacidades que influenciam as decisões na internacionalização e moderam os efeitos dos atributos institucionais e das diferenças institucionais nas estratégias das empresas. 
Como as Capacidades de Marketing Determinam a Escolha dos Modos de Entrada no Estrangeiro e a Seleção dos Países de Destino

\section{FUNDAÇÕES TEÓRICAS}

\section{Capacidades de marketing}

O conceito de capacidades de marketing (ou marketing capabilities, na sua designação em inglês) é relativamente recente nos estudos de marketing (Morgan, 2012; Kamboj \& Rahman, 2015). Segundo Day (1994), as capacidades de marketing são conjuntos complexos de habilidades e conhecimento acumulado, utilizados por meio de processos organizacionais que permitem a empresa coordenar as atividades e usar os seus ativos mais eficazmente. Assim, as capacidades de marketing são específicas a empresa. As capacidades de marketing referem-se à competência de a empresa usar os recursos disponíveis para realizar tarefas de marketing de modo a alcançar os resultados de marketing desejados (Day, 1994; Morgan et al., 2012). Em essência, as capacidades de marketing manifestamse nos processos que a empresa usa para definir, desenvolver, comunicar e agregar valor aos clientes, o que pode envolver a combinação, transformação e/ou implantação dos seus recursos disponíveis (Morgan et al., 2012; Morgan et al., 2018).

Quatro tipos de capacidades de marketing foram apresentadas e conceitualizadas por Morgan (2012) e Morgan et al. (2018) na seguinte tipologia: capacidades especializadas (que envolvem o planejamento, publicidade, gestão de produtos e gestão de canais), capacidades arquiteturais (envolvem a estratégia de marketing e implementação estratégica de marketing), capacidades multifuncionais (prendem-se com a gestão de marca, CRM, desenvolvimento de novos produtos) e as capacidades dinâmicas (que se referem a aprendizagem de mercado, reconfiguração de recursos e aprimoramento das demais capacidades). Cada uma destas capacidades de marketing é descrita em seguida.

As capacidades especializadas de marketing são as que estão no nível mais baixo ou, posto de outra forma, são as basilares e necessárias para conseguir operar. As capacidades especializadas referem-se aos processos específicos usados na organização para combinar e transformar recursos (Vorhies \& Morgan, 2005). Em essência, as capacidades especializadas se baseiam no marketing mix e na atuação simples da empresa nas atividades relacionadas com produtos, preços, comunicação e distribuição (Vorhies et al., 2009). As capacidades especializadas também podem existir a um nível que viabiliza a operação internacional da empresa. Nestes casos, além da gestão do marketing mix, inclui-se o conjunto de processos necessários para monitorar e obter informações relevantes sobre os mercados externos como sejam as características dos mercados, as necessidades e gostos dos clientes, etc. (Moller \& Anttila, 1987).

Num plano imediatamente superior estão as capacidades multifuncionais de marketing. Estas são capacidades relativamente mais complexas do que as capacidades especializadas na medida que já contemplam a integração de diversos recursos especializados. Ou seja, as capacidades multifuncionais reúnem vários recursos de marketing especializados e os combinam com entradas de recursos especializados em outras funções (Morgan, 2012). São exemplos de capacidades multifuncionais a gestão de marcas, gestão de relacionamento com clientes (CRM) e o desenvolvimento de novos produtos. Em todos estes casos a empresa precisa conseguir operar com mais do que capacidades isoladas.

As capacidades arquiteturais de marketing referem-se aos processos usados para selecionar, integrar e orquestrar vários recursos especializados e interfuncionais e suas entradas de recursos associadas (Ferreira, 2007). As capacidades arquiteturais de marketing são abrangentes dos processos relacionados ao planejamento envolvidos na seleção de objetivos estratégicos de marketing e na formulação de estratégias para alcançá-los (Morgan et al., 2003; Slotegraaf \& Dickson, 2004). Também incluem os processos relacionados à implementação, que facilitam a implantação de recursos múltiplos e inter-relacionados necessários para estabelecer estratégias de marketing (Capron \& Hulland, 1999; Vorhies \& Morgan, 2005). Capron e Hulland (1999) já colocavam uma visão dessas capacidades, como capacidades que englobam as práticas comerciais relacionadas ao desenvolvimento e à implementação da estratégia de marketing. As capacidades arquiteturais são compostas pelo planejamento estratégico de mercado, que é a habilidade da empresa de conceber estratégias de marketing apropriadas para alavancar os recursos e capacidades especializadas e multifuncionais disponíveis para a empresa para construir e manter a vantagem competitiva.

No patamar mais elevado estão as capacidades dinâmicas de marketing. As capacidades dinâmicas 
dizem respeito à capacidade da empresa de participar de um aprendizado baseado no mercado e usar o aprendizado acumulado para reconfigurar os recursos da empresa e aprimorar suas capacidades de maneiras que refletem o dinamismo do ambiente de mercado da empresa (Morgan, 2012). Lado et al. (1992) mencionaram competências avançadas da empresa, nomeando-as como competências de gestão e foco estratégico, como sendo fundamentais para obter vantagem competitiva sustentável. Fang e Zou (2009) compreendem as capacidades dinâmicas de marketing como a habilidade de resposta e a eficiência dos processos de negócios multifuncionais para criar e fornecer valor ao cliente face às mudanças do mercado. Numa ótica de capacidades dinâmicas, para proporcionar uma vantagem competitiva em ambientes dinâmicos, os recursos e capacidades da empresa precisam ser continuamente alterados, desenvolvidos e aprimorados (Lado et al., 1992; Fang \& Zou, 2009).

A tabela 1 , seguinte, sumaria a caracterização das capacidades de marketing. Num nível mais baixo as competências especializadas e as multifuncionais e num nível mais alto as competências dinâmicas e arquiteturais, destacando-se que diferentes capacidades envolvem diferentes formas de atuação.

Tabela 1. Capacidades de marketing

\begin{tabular}{|c|c|c|c|}
\hline $\begin{array}{c}\text { Capacidades de } \\
\text { marketing }\end{array}$ & Descrição & Exemplos & Referências \\
\hline Especializadas & $\begin{array}{l}\text { Habilidades de marketing } \\
\text { especializadas/funcionais, ou táticas, } \\
\text { na implementação de atividades de } \\
\text { mix de marketing }\end{array}$ & $\begin{array}{l}\text { - Gestão de produto } \\
\text { - Gestão de preço } \\
\text { - Gestão de canais de } \\
\text { distribuição } \\
\text { - Gestão da } \\
\text { comunicação } \\
\text { - Vendas } \\
\text { - Pesquisa de } \\
\text { mercado }\end{array}$ & $\begin{array}{l}\text { Goerzen et al. (2013); Chen, } \\
\text { Chen \& Zhou (2014); } \\
\text { Tan \& Sousa (2015); Gregory } \\
\text { et al. (2017) }\end{array}$ \\
\hline Multifuncionais & $\begin{array}{l}\text { Envolve a integração de conhecimentos } \\
\text { e habilidades em diferentes } \\
\text { subunidades funcionais, como } \\
\text { marketing, vendas e recursos humanos, } \\
\text { tornando-as multifuncionais por } \\
\text { natureza. }\end{array}$ & $\begin{array}{l}\text { - Gestão de marcas } \\
\text { - Gestão do } \\
\text { relacionamento com } \\
\text { clientes (CRM) } \\
\text { - Desenvolvimento } \\
\text { de novos produtos }\end{array}$ & $\begin{array}{l}\text { Blesa \& Ripolles (2008); } \\
\text { Bortoluzzi et al. (2014); } \\
\text { Eisend et al. (2016); Kaleka \& } \\
\text { Morgan (2017); Pham et al. } \\
\text { (2017) }\end{array}$ \\
\hline Arquiteturais & $\begin{array}{l}\text { Processos pelos quais as empresas } \\
\text { planejam combinações apropriadas } \\
\text { de conhecimentos disponíveis e } \\
\text { outros recursos para implantar em } \\
\text { seus mercados e executar essas } \\
\text { implantações, transformando-as em } \\
\text { ofertas de valor realizadas para o (s) } \\
\text { mercado (s) alvo (s) }\end{array}$ & $\begin{array}{l}\text { - Planejamento } \\
\text { estratégico de } \\
\text { mercado } \\
\text { - Implementação da } \\
\text { estratégia de } \\
\text { marketing }\end{array}$ & $\begin{array}{l}\text { Lages et al. (2009); Akdeniz et } \\
\text { al. (2010); Malik et al. (2012); } \\
\text { Morgan et al. (2012); } \\
\text { Wu (2013) }\end{array}$ \\
\hline Dinâmicas & $\begin{array}{l}\text { Associadas à reconfiguração de } \\
\text { recursos e ao aprimoramento das } \\
\text { capacidades de marketing atuais. }\end{array}$ & $\begin{array}{l}\text { - Aprendizagem de } \\
\text { marketing } \\
\text { - Reconfiguração de } \\
\text { recursos } \\
\text { - Aprimoramento de } \\
\text { capacidades }\end{array}$ & $\begin{array}{l}\text { Wilson \& Daniel (2007); } \\
\text { Lages et al. (2009); } \\
\text { Lu et al. (2010); } \\
\text { Evers et al. (2012) }\end{array}$ \\
\hline
\end{tabular}

Fonte: adaptado pelos autores de Morgan (2012) e Morgan, Feng \& Whitler (2018).

\section{Modos de entrada nos mercados externos}

A pesquisa sobre os modos de entrada no estrangeiro tem sido profícua desde os anos 1980 e revisões de literatura recentes podem ser consultadas em Andersen (1997), Morschett et al.
(2010), Klier et al. (2017) e Zhao, Ma e Yang (2017), entre outros. Não é relevante, para os nossos efeitos, revisar toda a literatura, mas importa distinguir os modos de entrada e a forma como podem ser influenciados pelas capacidades detidas pelas empresas que se internacionalizam quer para 
alavancar essas capacidades, quer pela busca por aumentar as capacidades nos mercados externos (Tallman \& Fladmoe-Lindquist, 2002; Ferreira, 2007).

A tipologia tradicional de modos de entrada apresentada em Root (1994) distingue exportação, licenciamento, parcerias (como joint-ventures), greenfield e aquisição. Também os trabalhos da escola de Upsalla (por exemplo, Johanson \& Vahlne, 1977) são úteis para distinguir a seleção dos modos de entrada em função da proximidade dos mercados, da experiência (ou conhecimento) e do comprometimento de recursos. Nesta linha, as empresas começam exportando e mais notoriamente para mercados próximos (cultural e economicamente próximos - usualmente países vizinhos) e à medida que acumulam conhecimento do mercado e do ambiente institucional e experiência de atuação no mercado envolvem-se em modos de entrada que exigem maior comprometimento de recursos (humanos, técnicos e financeiros) como sejam as joint-ventures ou operações de investimento direto no estrangeiro.

A exportação é a forma de atuação nos mercados externos que envolve menores riscos e que demanda menor comprometimento de recursos e menor conhecimento do mercado externo. As joint ventures envolvem a junção de ativos por duas ou mais empresas que constituem uma nova empresa de propriedade e controle conjuntos (Kogut \& Singh, 1988). Os ativos partilhados nas joint ventures podem ser físicos (por exemplo, redes de distribuição ou recursos alocados a P\&D) ou mais tácitos (conhecimento do mercado).

Modos de entrada que envolvem maior risco, comprometimento de recursos e, previsivelmente, maior conhecimento do mercado são os que requerem investimento direto no estrangeiro como os investimentos greenfield ou as aquisições de empresas incumbentes. Um investimento greenfield é um investimento inicial em novas instalações em que as empresas estrangeiras criam uma nova organização a partir do zero (Blesa \& Rippoles, 2008). Aquisições referem-se à compra de ações em uma empresa já existente, usualmente num montante suficiente para conferir controle (Kogut \& Singh, 1988) sobre as operações; embora uma aquisição possa envolver a compra do total do capital da empresa alvo (aquisição total) ou de apenas parte do capital (aquisição parcial). As vantagens das aquisições são bem conhecidas (ver Ferreira et al.
(2014) e Caiazza e Volpe (2015) para revisões da literatura). As aquisições permitem, por exemplo, que as empresas estrangeiras adquiram marcas locais e as combinem com suas capacidades específicas de marketing (Blesa \& Rippoles, 2008).

Esse processo ocorre porque há uma análise de risco feita por gestores que envolvem a distância geográfica, cultural, econômica e política entre os países envolvidos na internacionalização (Kraus et al., 2015). Suas empresas que se internacionalizam precisam ter a habilidade de diversificar, transferir ou mitigar os riscos (Müllner, 2016). Esse receio diante dos riscos faz com que o processo de entrada seja gradativo. É preferível nesse sentido opções que diminuam os riscos envolvidos a partir de iniciativas que vão aos poucos colocando a empresa que está se internacionalizando em contato com o país estrangeiro, suas instituições e suas organizações.

No entanto, a pesquisa sobre modos de entrada no estrangeiro tem explorado múltiplas dimensões externas à empresa, específicas a indústria e aos países de origem e destino (Shen et al., 2017). Considera-se também a relação entre as características da empresa e a escolha dos modos de entrada. Nesta linha das características específicas da empresa inclui-se a análise de aspectos que podem influenciar qual, ou quais, os modos de entrada adotados, como a experiência (Slangen \& Hennart 2008), os recursos (Morschett et al., 2008), e as capacidades (Tseng \& Lee 2010) das empresas investidoras. As capacidades detidas unidas à necessidade de manter a proteção dessas capacidades e de ativos específicos cuja monitorização seja difícil, aumenta a necessidade de modos de maior controle face a potenciais comportamentos oportunísticos de parceiros ou disseminação não intencional das capacidades (Hill \& Kim, 1988). Inclusive, a tecnologia que intermedia a transação entre empresa estrangeira e local parece também incrementar o controle da empresa mãe sobre as filiais de forma até a proporcionar melhor desempenho de marketing (Zhao \& Priporas, 2017). Isso leva a crer que há não somente uma relação entre a forma de controle e o método de entrada, mas também em como esses elementos estão relacionados ao sucesso do desempenho de marketing da companhia.

Ou seja, a internacionalização pode ser promovida, ou pelo menos facilitada, pelo fato da empresa deter vantagens específicas - neste caso as 
capacidades de marketing - mas a eficácia com que essas capacidades são utilizadas é função, também, dos modos de entrada adotados. Por outro lado, deficiências ou insuficiências nas capacidades podem conduzir as empresas a buscar formas de as aumentar, ou aprender (Barkema \& Vermeulen, 1998), nos mercados externos.Nesse sentido,conhecimentos e recursos podem ser acessados por meio de alianças de forma a potencializar o desempenho da internacionalização da empresa (Nakos et al., 2019). Para ilustrar isso, o estudo de Zacharakis (1997) já sugeria que as alianças são modos de entrada que as empresas podem usar para ultrapassar deficiências de capacidades e recursos, mas as formas de licenciamento e as jointventures podem ser adequadas para as empresas que detém menor conhecimento dos mercados locais.

\section{Seleção do país de destino}

A par da escolha dos modos de entrada, e de certa forma cumulativamente, a seleção dos mercados externos onde operar é, também, uma decisão estratégica fundamental na internacionalização (Papadopoulos \& Martín, 2011; Kim \& Aguilera, 2016). Para a seleção dos mercados externos presidem critérios de diferentes naturezas incluindose a avaliação da atratividade do mercado (ex., nível de renda, dotação populacional, acesso a recursos, perspectiva de crescimento, riscos, etc.). Outros fatores incluem a percepção de quão diferente é o mercado externo comparativamente ao mercado doméstico (a este propósito aprofundar sobre o conceito de distância institucional (Berry et al., 2010)) e a própria experiência internacional da empresa em operar nesse mercado ou em outros mercados externos (Johanson \& Vahlne, 1977; Takeuchi et al., 2005).

No entanto, a seleção do mercado externo também requer a análise do ajustamento das competências específicas da empresa às características do mercado e do ambiente institucional vigente. Ou seja, preside a seleção dos mercados a análise da adequação e da aplicabilidade das vantagens específicas da empresa, mas, também, a capacidade de as empresas transferirem know-how e conhecimento para as suas subsidiárias no estrangeiro (Dunning, 1980; Barkema \& Vermeulen, 1998). A transferência é especialmente relevante quando as empresas detêm algum tipo de conhecimento proprietário que procuram explorar dadas imperfeições de mercado (Hymer, 1976). A racionalidade clássica, remontando aos trabalhos de Hymer (1976) ou de Dunning (1988), e seu paradigma eclético, é que a internacionalização já subjaz que as empresas detêm uma vantagem que podem explorar no estrangeiro. Atualmente, essa racionalidade mantém-se, mas estende-se a observação da estratégia de longo prazo da empresa e do ambiente institucional dos países (e como eles diferem) na sua influência sobre a seleção da localização.

Alguns estudos têm focado como as vantagens específicas das empresas, as suas motivações estratégicas, e as suas capacidades, podem moderar eventuais impactos do ambiente institucional e diferenças institucionais na escolha das localizações pelas empresas (Dunning \& Lundan 2008). Por exemplo, as capacidades tecnológicas (Henisz \& Macher, 2004), experiência prévia em lidar com ambientes institucionalmente distintos que Holburn e Zelner (2010) designaram por capacidades políticas, a internacionalizar, ou, pelo menos a não evitar, ambientes institucionais instáveis ou mais distintos do seu país de origem. Em suma, a forma como as instituições e atributos locais influenciam a escolha da localização pelas empresas depende das suas características e capacidades específicas, das motivações estratégicas e da transferência das capacidades para os mercados externos (ver Kim \& Aguilera, 2016).

\section{DESENVOLVIMENTO CONCEITUAL}

\section{Capacidades de marketing e seleção dos modos de entrada}

As capacidades dinâmicas de internacionalização das empresas (Pinho \& Prange, 2016) são um dos elementos que influenciam o sucesso de suas empreitadas internacionais. Uma dessas capacidades é a sua capacidade de marketing. Inclusive, a forma como as empresas pretendem entrar e operar nos mercados externos - usualmente designada por modos de entrada no estrangeiro - pode ser influenciada pelas capacidades de marketing detidas. Modos de entrada, como exportações e licenciamento, estão associados a níveis mais baixos de risco e de controle sobre as estratégias operacionais e de marketing envolvidas na internacionalização (Blesa \& Rippoles, 2008). Com frequência, e pelo menos em fases iniciais, os esforços de exportação das empresas compreendem um único produto ou linha de produtos (Cavusgil \& Zou, 1994). Ainda, como já evidenciado, muitas empresas são motivadas a exportar por um agente de 
Como as Capacidades de Marketing Determinam a Escolha dos Modos de Entrada no Estrangeiro e a Seleção dos Países de Destino

mudança externo (por exemplo, um cliente estrangeiro), algumas simplesmente aproveitam as oportunidades de exportação que surgem sem um objetivo evidente em mente, enquanto outras são motivadas a iniciar a exportação com um objetivo internacional mais claro (Root, 1994). Assim, empresas que optam pela exportação para entrar em mercados internacionais, acabam não necessitando possuir capacidades de marketing tão desenvolvidas para terem sucesso no país de destino.

A entrada nos mercados estrangeiros por meio de parcerias pode visar colmatar lacunas existentes. As parcerias são um modo de entrada que encerra menor risco, controle partilhado e menor envolvimento direto de recursos que as formas de investimento direto. Por exemplo, Lu e Beamish (2001) notaram que as alianças com parceiros que detém conhecimento do mercado local são uma estratégia de obviar a insuficiências na base de recursos e capacidades detidas pelas empresas. As parcerias, como as alianças ou joint-ventures, ultrapassando deficiências nas capacidades, aumentam a probabilidade de sucesso na internacionalização (Zacharakis, 1997). Também Davidson (1980) notou que quando as empresas não detêm conhecimento do mercado externo podem preferir modos de entrada como as parcerias ou acordos de licenciamento, em vez de investimento em subsidiárias wholly owned. Muitas vezes a parceria se dá com um parceiro do país de destino, que já tem capacidades de marketing (arquiteturais e dinâmicas) bem desenvolvidas para competir no mercado local.

Isso possibilita que empresas que possuem apenas capacidades de marketing mais básicas desenvolvidas, atuar no mercado internacional, uma vez que o parceiro local tem capacidades de marketing suficientes e adequadas a viabilizar o negócio.

Em essência, modos de entrada de baixo comprometimento como sejam as exportações e modos que envolvem a partilha, como sejam as jointventures, requerem menores capacidades de marketing por parte da empresa, em uma decisão de atuar no mercado internacional, em comparação com modos de entrada de maior comprometimento. Isso se faz visível, quando pequenas empresas, até mesmo iniciantes no mercado, muitas vezes de produtos de baixo valor agregado duráveis (ex.: fones de ouvido, mouses, utensílios de cozinha, calçados) ou não duráveis (ex.: salgadinhos, doces em geral, commodities), cujas marcas e produtos não se diferenciam dos demais, optam pela exportação (ou, por vezes, uma joint-venture) por uma simples oportunidade de negócio, com único objetivo de ampliação de mercado. Em suma, argumentamos que as empresas que possuem apenas capacidades de marketing de níveis mais baixos conduzam a preferência por modos de entrada como sejam a exportação ou formas de parceria como as jointventures.

Proposição 1. Empresas com apenas as capacidades de marketing especializadas e multifuncionais desenvolvidas, tenderão a escolher entrar nos mercados externos por meio de exportação e joint-venture, do que por aquisições ou investimentos greenfield.

As capacidades arquiteturais de marketing são definidas como o processo pelo qual as empresas planejam combinações de conhecimentos disponíveis com outros recursos para entrar em novos mercados e transformá-los em oferta de valor ao mercado alvo (Vorhies \& Morgan, 2005). Novos modelos de desenvolvimento tecnológico em empresas multinacionais tratam as capacidades arquiteturais como essenciais para a coordenação de esforços tecnológicos além das fronteiras do país de origem. O conhecimento arquitetural adquirido na gestão de corporações domésticas pode ser ampliado para gerir de forma mais eficaz as suas operações nos mercados internacionais (Tallman \& FladmoeLindquist, 2002; Ferreira, 2007).

De modo similar, o conceito de capacidades dinâmicas postula que, uma vez que os mercados são dinâmicos, não basta apenas a simples heterogeneidade nos recursos das empresas, mas sim as capacidades pelas quais seus recursos são adquiridos e implantados, adaptados ao o ambiente de mercado da firma, explicando uma melhor adaptação em mercados distintos, complexos e dinâmicos (Teece, Pisano \& Shuen, 1997). As capacidades são dinâmicas quando permitem que a empresa implemente novas estratégias para refletir as condições mutantes do mercado, combinando e transformando os recursos disponíveis de maneiras novas e diferentes (Teece et al., 1997). As capacidades dinâmicas de marketing podem ser concebidas como tendo três elementos principais: aprendizado de mercado, reconfiguração de recursos e aprimoramento de capacidade. (Morgan, 2012). Há evidência que, em países em desenvolvimento, as 
capacidades dinâmicas de marketing contribuem diretamente para o crescimento das vendas das subsidiárias no estrangeiro (Konwar et al., 2017).

A forma como os recursos e capacidade das empresas influenciam os modos de entrada não é clara e, por exemplo, as empresas podem preferir modos de entrada que lhes concedem proteção de propriedade proprietária ou que diminuam o risco de disseminação das suas capacidades distintivas (Hill \& Kim, 1988). Quando os custos de transação aumentam para proteção, então é possível que prefiram modos que envolvem a internalização, por meios que envolvem o controle das operações (subsidiárias no exterior detidas a 100\%). Assim, apesar das formas de exportação serem as que envolvem menor comprometimento de recursos e menores riscos, se a empresa detêm ativos ou capacidades que precisa proteger, a exportação é uma forma que não concede proteção do oportunismo (por exemplo de distribuidores), nem as parcerias protegem dos oportunismos potenciais dos parceiros. As joint-ventures envolvem a partilha com parceiros e, potencialmente, custos de transação mais altos, mas podem ser modos justificáveis quando a empresa detém algumas capacidades mas tem insuficiências, ou algum tipo de ativo que não é apropriado, para entrar num mercado externo (Hennart \& Park, 1993). Então, se as empresas encaram riscos mais elevados ou insuficiências difíceis de suprir por meio de parcerias, podem contemplar modos de investimento direto estrangeiro por meio de operações greenfield ou aquisição de incumbentes. Previsivelmente, as insuficiências não serão ao nível de ativos básicos mas sim ao nível de ativos mais complexos que envolvem a arquitetura ou a capacidade de integrar operações. Por exemplo, as aquisições podem permitir que a empresa adquirente adquira marcas locais e as combine com o seu portefólio de marcas usando as suas capacidades de marketing - esta habilidade pode inclusive ser crucial como forma de adaptação local (Hennart \& Park, 1993).

Assim, argumentamos que as empresas que detém capacidades arquiteturais de marketing estão melhor preparadas para se internacionalizarem por meio de aquisições, sendo que possuem mais experiência e conhecimento em planejamento estratégico de marketing e em implementação das estratégias de marketing. Dadas as características das capacidades arquiteturais e dinâmicas, propomos que apenas alcançando esse nível de desenvolvimento das capacidades de marketing, as empresas podem ser bem-sucedidas em sua internacionalização por meio de aquisição ou por greenfield. Estes são dois modos de entrada em que a empresas conseguem absorver conhecimentos de novos mercados e adaptar suas estratégias com mais facilidade e agilidade sem necessitar de parceiros e sem necessitar adquirir recursos por meio de aquisições. Como notaram Capron \& Hulland (1999), uma capacidade superior de marketing pode ser uma fonte de vantagem competitiva de uma empresa. Por exemplo, a Procter \& Gamble (P\&G) mostrou a capacidade de combater seus principais concorrentes em muitos mercados devido a deter marcas fortes e força de vendas, mas, também, pela sua capacidade de adquirir conhecimento e se adaptar a diferentes contextos de mercado. A P\&G tende a realizar aquisições de empresas e marcas já instaladas ou realizar investimentos greenfield em novos processos de expansão internacional.

Em suma, argumentamos que empresas com capacidades de marketing arquiteturais e dinâmicas bem desenvolvidas tenderão a preferir as aquisições ou os investimentos greenfield.

Pelo menos em parte, a escolha do modo de entrada é determinada por não necessitarem parceiros locais que façam o aporte de conhecimentos e competências que já são detidos internamente ou que podem ser construídos internamente, enquanto permitem maior controle sobre as operações no estrangeiro que reduzem o risco de difusão não intencional das capacidades (Hill \& Kim, 1988).

Proposição 2. Empresas com capacidades de marketing arquiteturais e dinâmicas desenvolvidas tenderão a escolher entrar nos mercados externos por meio de aquisições ou operações greenfield, do que por exportação ou joint-ventures.

\section{Capacidades de marketing e seleção dos países de destino}

As capacidades de marketing também podem ser um preditor da escolha dos mercados para onde internacionalizar; ou seja, da seleção dos mercados externos. A seleção dos mercados externos influencia a configuração operacional das empresas porque determina aspectos tão diversos como a produção, gestão financeira, elementos organizacionais e gerenciais que precisam ser ajustados não apenas às operações no estrangeiro, mas, também, atendendo 
à organização interna das próprias multinacionais. As decisões sobre quais mercados internacionais entrar depende de aspectos diversos como os produtos a comercializar no exterior, ou a exportar, e de como as capacidades detidas permitirão competir, e não são apenas dependentes da atratividade econômica do mercado alvo. As avaliações das habilidades, capacidades e objetivos da empresa devem ser consideradas. A visão baseada em capacidades sugere que o sucesso nos mercados externos dependerá crucialmente de quão bem as empresas são capazes de aprender (Barkema \& Vermeulen, 1998) e desenvolver recursos e capacidades distintas (Ferreira, 2007; Gaur et al., 2014).

Empresas de países mais desenvolvidos são propensas a investir em países menos desenvolvidos para proteger seus mercados, aos quais anteriormente forneciam através de exportações. Essas empresas produzem e exportam novos produtos, de alta qualidade, com altos insumos de marketing e alta tecnologia que não são muito sensíveis à concorrência de preços (Lecraw, 1977). As empresas multinacionais de países em desenvolvimento tendem a ser menos competitivas que as de países desenvolvidos, em parte porque sofrem a desvantagem de operar em países de origem com instituições menos desenvolvidas (Cuervo-Cazurra \& Genc, 2008). Em contraste, as empresas multinacionais de países mais desenvolvidos tendem a ter vantagens de propriedade mais fortes em áreas como branding e publicidade, tecnologia, entre outras (CuervoCazurra, 2012). Em síntese, argumentamos que empresas que decidirem atuar em países com nível de desenvolvimento idêntico ou inferior ao seu país de origem, não necessitam deter capacidades de marketing de nível mais alto. Por exemplo, há evidência que as multilatinas são mais propensas a iniciar sua internacionalização por países mais próximos cultural, econômica e geograficamente. Pelo menos em parte a escolha de países próximos é função da similaridade institucional e em parte da capacidade de alavancar recursos e capacidades para esses mercados - como parecem ser os casos de empresas como a Salinas (México), Techint (Argentina), Gerdau (Brasil) e Odebrecht (Brasil) (Cuervo-Cazurra, 2008) que conseguem alavancar o conhecimento acumulado nos seus países de origem em mercados similares da América Latina.

Proposição 3. Empresas com apenas capacidades de marketing especializadas e multifuncionais desenvolvidas, tenderão a escolher países com nível de desenvolvimento igual ou inferior ao do seu país de origem.

Embora o investimento direto estrangeiro externo possa ser motivado por as empresas buscarem superar desvantagens competitivas resultantes de ineficiências e vazios institucionais do seu país de origem (numa lógica que Stoian e Mohr (2016) e Stal e Cuervo-Cazurra (2011) designaram por "escapism" para referir a internacionalização das empresas como fuga as ineficiências do mercado doméstico), a realização de operações no estrangeiro, e as operações de IDE especificamente, requer a detenção de vantagens competitivas que as auxiliem a superar os custos e desvantagens de ser estrangeiro (Zaheer, 1995). Nestes casos, as características locais dos mercados e das instituições podem ser indutoras dos modos de entrada a adotar. Ou seja, estas empresas podem necessitar acessar capacidades de marketing não detidas individualmente e inclusive mudar a sua estratégia de exportação para optarem por entrar no exterior usando formas de investimento estrangeiro direto em países mais desenvolvidos (Gaur et al., 2014; Stoian \& Mohr, 2016).

Há evidências, pelo menos a um nível casuístico, que algumas empresas originárias de economias emergentes começaram a partir dos anos 2000, foram capazes de desenvolver um leque de capacidades nos seus mercados domésticos que começaram a explorar internacionalmente e se tornaram líderes nas suas indústrias, como a fabricante de aviões brasileira Embraer ou a fabricante de equipamentos de telecomunicações chinesa Huawei, e suas aquisições ousadas em economias avançadas, como a compra da produtora de aço holandesa Corus pelo conglomerado indiano Tata ou a aquisição da britânica Cemex pela empresa mexicana RMC (Cuervo-Cazurra, 2012). As multinacionais de países em desenvolvimento, diferente das multinacionais de países desenvolvidos, se internacionalizam ao mesmo tempo em que obtêm novos recursos e capacidades por meio de alianças ou aquisições de empresas para melhorar suas capacidades desenvolvidas no mercado doméstico (Kumaraswamy et al., 2012). Assim, o modelo tradicional no qual a multinacional usa recursos e capacidades existentes para expandir no exterior é modificado. Quando as empresas de países em desenvolvimento, aumentam suas capacidades de marketing, competindo em alto nível no seu país 
de origem, passam a optar por atuar não somente em países menos desenvolvidos, ou equivalentes ao seu país de origem, mas percebem a possibilidade de atuação em países mais desenvolvidos, mesmo que algumas capacidades ainda precisem ser mais desenvolvidas no decorrer do processo de internacionalização.

Então, os mercados mais desenvolvidos tendem a ter indústrias mais complexas e mercados mais eficientes, onde a rivalidade entre as empresas é mais acirrada (em parte devido ao modelo competitivo mais sofisticado em que as empresas detém uma imagem de marca ou tecnologia superior), provavelmente levarão a que apenas as empresas que possuam capacidades de marketing de níveis superiores entrem nesses mercados. Ou seja, o nível de desenvolvimento dos países receptores é um determinante de quais as capacidades de marketing que são necessárias para conseguir bons níveis de desempenho. Face ao exposto anteriormente, propomos que:

Proposição 4. Empresas com capacidades de marketing arquiteturais e dinâmicas desenvolvidas tenderão a escolher países mais desenvolvidos que o seu país de origem.

A figura 1 seguinte ilustra as capacidades de marketing como uma pirâmide em que no topo estão as capacidades mais sofisticadas e na base as mais especializadas e funcionais, as quais correspondem diferentes escolhas de mercados estrangeiros (escolha de localização) e de modos de serviços, ou modos de entrada, desses mercados.

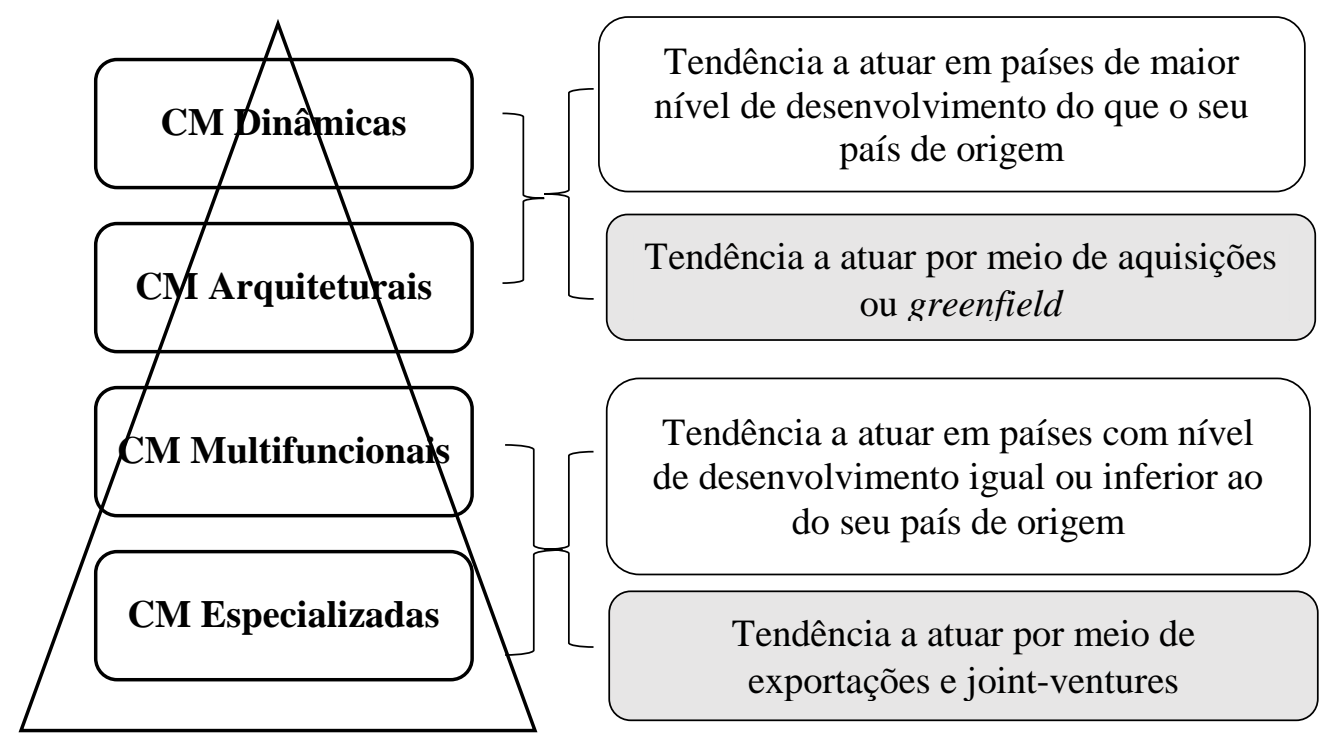

Figura 1. Capacidades de marketing na internacionalização: mercados e modos de entrada Fonte: Conceitualização dos autores.

\section{DISCUSSÃO}

Neste estudo propomos analisar as capacidades de marketing observando a sua influência em duas decisões fundacionais das operações internacionais das empresas: a escolha do modo de entrada no estrangeiro e a seleção dos mercados externos para onde internacionalizar. Este estudo complementa, assim, pesquisa já existente sobre as capacidades de marketing (Moller \& Antilla, 1987; Vorhies \& Morgan, 2005; Blesa \& Rippoles, 2008; Morgan, 2012; Evers et al., 2012; Gregory et al., 2017; Pham et al., 2017; Morgan et al., 2018), inclusive na sua conceitualização, mas chamando por mais pesquisas que possam entender como as diferentes capacidades podem influenciar duas decisões estratégicas fundamentais em negócios internacionais: a escolha dos modos de entrada e dos países para onde expandir. De algum modo este é um contributo para entender quais as vantagens específicas a empresa e como estas determinam as ações (Kotha et al., 2001).

Pertinente, poderá ser, por exemplo, o aprofundamento de como as capacidades de marketing são aumentadas na internacionalização, dado que o nosso foco é primordialmente numa perspectiva de alavancagem de capacidades já detidas. Esta linha é tão mais relevante quanto a pesquisa sobre vantagens específicas a empresa tem 
mostrado que estas vantagens específicas são fundacionais para a internacionalização (Kotha et al., 2001) e que a captura e novas capacidades ou o desenvolvimento das existentes pode ser um papel relevante para as subsidiárias (Birkinshaw et al., 1998). No entanto, entre as vantagens específicas das empresas não tem sido incluída a análise de competências de marketing e especialmente seguindo a distinção nas quatro dimensões de capacidades especializadas, multifuncionais, dinâmicas e arquiteturais.

Espera-se que a presente proposta possa oferecer novas bases para os estudos sobre o país de destino na internacionalização e suas respectivas estratégias de entrada. Isso significa que além do distanciamento considerado em estudos sobre o país de destino como a distância geográfica, cultural, econômica e política (Kraus et al., 2015), há também a consideração das capacidades de marketing. Ao se propor aqui que essas capacidades devem levar em consideração também variáveis e construtos sob a perspectiva das capacidades de marketing, espera-se poder ampliar as possibilidades de compreensão desse fenômeno do ponto de vista teórico. Com isso, a expectativa é que o conhecimento construído na área tenha especificidades, variáveis e construtos que incrementem o poder explicativo dos modelos que consideram a escolha do país de destino e a estratégia do método de entrada.

\section{CONCLUSÕES}

O argumento fundamental desenvolvido neste trabalho conceitual é que as empresas detêm diferentes capacidades de marketing e que estas vantagens podem ser instrumentais para o desempenho nos mercados externos. No entanto, é necessário o ajustamento entre as capacidades e quer o modo de entrada selecionado, quer o local onde as vantagens concedidas pelas capacidades são melhor alavancadas. Neste sentido, podemos sumariar a nossa proposta como: empresas com apenas capacidades de marketing a um nível mais basilar - capacidades especializadas e/ou multifuncionais - mais provavelmente tenderão a escolher modos de entrada de menor envolvimento de recursos e a internacionalizar-se para países mais próximos - o que está de acordo com a ideia de incrementalismo na internacionalização da escola de Upsalla. Em contraste, empresas com capacidades de marketing mais sofisticadas - capacidades arquiteturais e/ou dinâmicas - também conseguem ser bem-sucedidas por meio de modos de entrada de maior comprometimento de recursos como as aquisições e/ou operações greenfield, e mesmo em países mais distantes.

Esta argumentação é sustentada em que os diferentes modos de entrada no exterior têm diferentes níveis de complexidade. Assim, ao escolher modos de entrada mais complexos - como as aquisições ou greenfield - as empresas necessitam deter capacidades de marketing mais sofisticadas que consistem e concedem maior facilidade de aprendizagem, capacidade para reconfigurar e aprimorar os recursos detidos, e melhor desempenho no planejamento e implementação das estratégias de marketing como capacidades de marketing arquiteturais e dinâmicas.

Essa proposta oferece novas perspectivas para a construção de modelos de entrada em novos mercados internacionais considerando as capacidades de marketing das empresas. A partir disso, novos conhecimentos sobre os meios de entrada a partir das capacidades de marketing podem complementar as atuais teorias. A proposta desse ensaio pode oferecer maior especificidade aos estudos que contemplam o distanciamento entre os países para compreender métodos de entrada em iniciativas de internacionalização considerando também as capacidades de marketing. Oferece-se assim maiores perspectivas para a compreensão empírica dos fenômenos de meios de entrada em países com distanciamento também do ponto de vista de marketing.

Do ponto de vista gerencial, há uma proposta sobre elementos relacionados às capacidades de marketing que o gestor pode refletir melhor na sua decisão sobre onde internacionalizar. Isto é, essa escolha de qual país de destino se pretende internacionalizar pode ser feita a partir da análise comparativa das capacidades de marketing usualmente existentes no seu país de origem em relação ao seu país de destino. Há uma orientação do que ele pode fazer em termos de escolha de país ao qual vai se internacionalizar e a forma como a parceria internacional será conduzida a partir da análise comparativa de capacidade de marketing entre empresas de diferentes países.

A assunção é que, pelo menos em parte, para ter sucesso na atuação em países mais desenvolvidos, em relação ao país de origem, é necessário que a empresa tenha capacidades de marketing (mais 
especificamente, arquiteturais e dinâmicas) bem desenvolvidas. A evidência reside na necessidade de conseguir competir em indústrias mais complexas e com níveis superiores de sofisticação. Em contraponto, para operar em países de nível de desenvolvimento similar ou inferior, capacidades de marketing especializadas ou multifuncionais desenvolvidas serão suficientes (talvez não ótimas) para a escolha de atuação internacionais nestes países, dada a semelhança dos mercados, instituições e até mesmo, do patamar de desenvolvimento da concorrência.

\section{Extensões para pesquisa futura}

Pesquisa futura pode evoluir em várias direções. Por exemplo, dado que a conceitualização das capacidades de marketing ainda é relativamente recente (Morgan, 2012) seria relevante identificar e/ou construir métricas para a sua mensuração (Kamboj \& Rahman, 2015). Estas métricas permitiriam aferir os quatro níveis de capacidades de marketing utilizados - especializadas, multifuncionais, arquiteturais e dinâmicas - e o desenvolvimento de estudos empíricos para o teste das proposições avançadas prevendo o impacto das diferentes capacidades de marketing sobre os modos de entrada e seleção do mercado de destino, entre outras variáveis relevantes nas estratégias das empresas.

Numa perspectiva de negócios internacionais e internacionalização de empresas, a conceitualização adicional das capacidades de marketing, talvez mais especialmente as capacidades arquiteturais e as dinâmicas, exige entender até que ponto essas competências podem ser alavancadas nas operações no estrangeiro. Isto envolve entender como as capacidades de marketing são valiosas quando as empresas precisam operar em localizações com ambiente institucional, cultural, econômico, legal e infra estrutural diferente daquele onde foram inicialmente desenvolvidas - ver Ferreira (2007) a este respeito.

Pesquisa adicional também é necessária para o entendimento de quais as capacidades fundamentais para operar em mercados mais e menos desenvolvidos. Estudos com o teste de hipóteses relacionando as diferentes capacidades de marketing com o nível de desenvolvimento do país de destino podem evidenciar quais são as capacidades fundamentais. Por exemplo, é possível que para operar em países institucionalmente menos desenvolvidos seja suficiente deter capacidades de nível mais baixo (especializadas e multifuncionais), como propusemos e para operar em mercados mais sofisticados sejam necessárias capacidades arquiteturais e dinâmicas. No entanto, as diferenças institucionais entre países e o sentido dessas diferenças podem, previsivelmente, alterar o valor das capacidades para cada tipo de diferença institucional (ver, uma tipologia de distâncias institucionais em Berry et al. (2010)).

Numa linha complementar, importa notar o questionamento de Erramilli et al. (1997) se as vantagens específicas as empresas serão também específicas a localização. Deste modo, as capacidades de marketing desenvolvidas num contexto nacional podem não ser transferíveis para outras localizações. Por exemplo, as capacidades de marketing desenvolvidas por empresas brasileiras no seu mercado doméstico podem não ser transferíveis para outras economias emergentes como a China ou Índia, denotando que seriam, efetivamente, capacidades de marketing específicas a sua localização original. Então, estudos futuros podem explorar se, e até que ponto, as competências específicas das empresas, onde se incluem as capacidades de marketing, são utilizáveis pelas subsidiárias no estrangeiro ou se são acumuláveis (ou aprendidas) pelas subsidiárias no estrangeiro e depois passíveis de ser transferidas internamente (Birkinshaw, Hood \& Jonsson, 1998).

\section{REFERÊNCIAS}

Akdeniz, M., Gonzalez-Padron, T. \& Calantone, R. (2010). An integrated marketing capability benchmarking approach to dealer performance through parametric and nonparametric analyses. Industrial Marketing Management, 39(1), 150-160.

Anand, J., \& Delios, A. (2002). Absolute and relative resources as determinants of international acquisitions. Strategic Management Journal, 23(2), 119-134.

Andersen, O. (1997). Internationalization and market entry mode: A review of theories and conceptual frameworks. Management International Review, 37(2), 27-42.

Barkema, H., \& Vermeulen, F. (1998). International expansion through start-up or acquisition: A learning perspective. Academy of Management Journal, 41(1), 7-26. 
Berry, H., Guillén, M., \& Zhou, N. (2010). An institutional approach to cross-national distance. Journal of International Business Studies, 41(9), 1460-1480.

Birkinshaw, J., Hood, N., \& Jonsson, S. (1998). Building firm-specific advantages in multinational corporations: the role of subsidiary initiative. Strategic Management Journal, 19(3), 221242.

Blesa, A., \& Ripolles, M. (2008). The influence of marketing capabilities on economic international performance. International Marketing Review, 25(6), 651-673.

Bortoluzzi, G., Chiarvesio, M., Di Maria, E., \& Tabacco, R. (2014). Exporters moving toward emerging markets: A resource-based approach. International Marketing Review, 31(5), 506-525.

Brouthers, L., Mukhopadhyay, S., Wilkinson, T., \& Brouthers, K. (2009). International market selection and subsidiary performance: A neural network approach. Journal of World Business, 44(3), 262-273.

Caiazza, R., \& Volpe, T. (2015). M\&A process: A literature review and research agenda. Business Process Management Journal, 21(1), 205-220.

Capron, L., \& Hulland, J. (1999). Redeployment of brands, sales forces, and general marketing management expertise following horizontal acquisitions: A resource-based view. Journal of Marketing, 63(2), 41-54.

Cavusgil, S., \& Knight, G. (2015). The born global firm: An entrepreneurial and capabilities perspective on early and rapid internationalization. Journal of International Business Studies, 46(1), 3-16.

Cavusgil, S., \& Zou, S. (1994). Marketing strategyperformance relationship: An investigation of the empirical link in export market ventures. Journal of Marketing, 58(1), 1-21.

Chen, S. (2008). The motives for international acquisitions: Capability procurements, strategic considerations, and the role of ownership structures. Journal of International Business Studies, 39(3), 45471.

Chen, X., Chen, A., \& Zhou, K. (2014). Strategic orientation, foreign parent control, and differentiation capability building of international joint ventures in an emerging market. Journal of International Marketing, 22(3), 30-49.

Cuervo-Cazurra, A. (2012). Extending theory by analyzing developing country multinational companies: Solving the Goldilocks debate. Global Strategy Journal, 2(3), 153-167.

Cuervo-Cazurra, A., \& Genc, M. (2008). Transforming disadvantages into advantages: Developing-country MNEs in the least developed countries. Journal of International Business Studies, 39(6), 957-979.

Day, G. (1994). The capabilities of market-driven organizations. Journal of Marketing, 58, 37-52.

Davidson, W. (1980). The location of foreign direct investment activity: country characteristics and experience effects. Journal of International Business Studies, 11, 9-22.

Dunning, J. (1980). Toward an eclectic theory of international production: Some empirical tests. Journal of International Business Studies, 11(1), 9-31.

Dunning, J., \& Lundan, S. (2008). Multinational enterprises and the global economy. Cheltenham: Edward Elgar.

Dutta, S., Narasimhan, O., \& Rajiv, S. (1999). Success in high-technology markets: Is marketing capability critical? Marketing Science, 18(4), 547-568.

Eisend, M., Evanschitzky, H., \& Calantone, R. (2016). The relative advantage of marketing over technological capabilities in influencing new product performance: the moderating role of country institutions. Journal of International Marketing, 24(1), 41-56.

Erramilli, M., Agarwal, S., \& Kim, S. (1997). Are firm-specific advantages location-specific too? Journal of International Business Studies, 28(4), 735-757.

Evers, N., Andersson, S., \& Hannibal, M. (2012). Stakeholders and marketing capabilities in international new ventures: Evidence from Ireland, Sweden and Denmark. Journal of International Marketing, 20(4), 46-71.

Fang, E., \& Zou, S. (2009). Antecedents and consequences of marketing dynamic capabilities in 
international joint ventures. Journal of International Business Studies, 40(5), 742-761.

Ferreira, M. (2007). Building and leveraging knowledge capabilities through cross-border acquisitions. In Tallman, S. (Org.). New Generations in International Strategy. New York, NY: Edward Elgar Publishing, Ltd.

Ferreira, M., Santos, J., Almeida, M., \& Reis, N. (2014). Mergers \& acquisitions research: A bibliometric study of top strategy and international business journals, 1980-2010. Journal of Business Research, 67(12), 2550-2558.

Gaur, A., Kumar, V., \& Singh, D. (2014). Institutions, resources, and internationalization of emerging economy firms. Journal of World Business, 49(1), 12-20.

Goerzen, A., Asmussen, C., \& Nielsen, B. (2013). Global cities and multinational enterprise location strategy. Journal of international business studies, 44(5), 427-450.

Gregory, G., Ngo, L., \& Karavdic, M. (2017). Developing e-commerce marketing capabilities and efficiencies for enhanced performance in business-tobusiness export ventures. Industrial Marketing Management, in press.

Henisz, W., \& Macher, J. (2004). Firm-and country level trade-offs and contingencies in the evaluation of foreign investment: the semiconductor industry, 19942002. Organization Science, 15, 537-554.

Hennart, J., \& Park, Y. (1993). Greenfield vs. acquisition: The strategy of Japanese investors in the United States. Management Science, 39(9), 1054-1070.

Hennart, J., \& Slangen, A. (2015). Yes, we really do need more entry mode studies! A commentary on Shaver.Journal of International Business Studies, 46(1), 114-122.

Hill, C., \& Kim, W. (1988). Searching for a dynamic theory of the multinational enterprise: A transaction cost model. Strategic Management Journal, 9, 93194.

Holburn, G., \& Zelner, B. (2010). Political capabilities, policy risk, and international investment strategy: Evidence from the global electric power generation industry. Strategic Management Journal, 31, 1290-1315.
Hymer, S. (1976). The international operations of national firms: A study of direct foreign investment. Cambridge, MA: MIT Press.

Johanson, J., \& Vahlne, J. (1977). The internationalization process of the firm: A model of knowledge development and increasing foreign market commitments. Journal of International Business Studies, 8(1), 23-32

Kaleka, A., \& Morgan, N. (2017). How marketing capabilities and current performance drive strategic intentions in international markets. Industrial Marketing Management. Online first doi: 10.1016/j.indmarman.2017.02.001.

Kamboj, S., \& Rahman, Z. (2015). Marketing capabilities and firm performance: Literature review and future research agenda. International Journal of Productivity and Performance Management, 64(8), 1041-1067.

Kim, J., \& Aguilera, R. (2016). Foreign location choice: Review and extensions. International Journal of Management Reviews, 18(2), 133-159.

Klier, H., Schwens, C., Zapkau, F., \& Dikova, D. (2017). Which resources matter how and where? A meta-analysis on firms' foreign establishment mode choice. Journal of Management Studies, 54(3), 304339.

Kogut, B., \& Singh, H. (1988). The effect of national culture on the choice of entry mode. Journal of International Business Studies, 19(3), 411-432.

Konwar, Z., Papageorgiadis, N., Ahammad, M., Tian, Y., McDonald, F., \& Wang, C. (2017). Dynamic marketing capabilities, foreign ownership modes, sub-national locations and the performance of foreign affiliates in developing economies. International Marketing Review, 34(5), 674-704.

Kotabe, M., Srinivasan, S., \& Aulakh, P. (2002). Multinationality and firm performance: The moderating role of R\&D and marketing capabilities. Journal of International Business Studies, 33(1), 79-97.

Kotha, S., Rindova, V., \& Rothaermel, F. (2001). Assets and actions: Firm-specific factors in the internationalization of US Internet firms. Journal of International Business Studies, 32(4), 769-791.

Krasnikov, A., \& Jayachandran, S. (2008). The relative impact of marketing, research-and-development, and 
operations capabilities on firm performance. Journal of marketing, 72(4), 1-11.

Kraus, S., Ambos, T. C., Eggers, F., \& Cesinger, B. (2015). Distance and perceptions of risk in internationalization decisions. Journal o Business Research, 68(7), 1501-1505.

Kumaraswamy, A., Mudambi, R., Saranga, H., \& Tripathy, A. (2012). Catch-up strategies in the Indian auto components industry: Domestic firms' responses to market liberalization. Journal of International Business Studies, 43(4), 368-395.

Lado, A., Boyd, N., \& Wright, P. (1992). A competency-based model of sustainable competitive advantage: Toward a conceptual integration. Journal of Management, 18(1), 77-91.

Lages, L., Silva, G., \& Styles, C. (2009). Relationship capabilities, quality, and innovation as determinants of export performance. Journal of international Marketing, 17(4), 47-70.

Lecraw, D. (1977). Direct investment by firms from less developed countries. Oxford Economic Papers, 29(3), 442-457.

Lee, R., \& Zhou, K. (2012). Is product imitation good for firm performance? An examination of product imitation types and contingency factors. Journal of International Marketing, 20(3), 1-16.

Lu, J., \& Beamish, P. (2001). The internationalization and performance of SMEs. Strategic Management Journal, 22, 565-586.

Lu, Y., Zhou, L., Bruton, G., \& Li, W. (2010). Capabilities as a mediator linking resources and the international performance of entrepreneurial firms in an emerging economy. Journal of International Business Studies, 41(3), 419-436.

Malik, A., Sinha, A., \& Blumenfeld, S. (2012). Role of quality management capabilities in developing market-based organisational learning capabilities: Case study evidence from four Indian business process outsourcing firms. Industrial Marketing Management, 41(4), 639-648.

Meyer, K., Wright, M., \& Pruthi, S. (2009). Managing knowledge in foreign entry strategies: A resource-based analysis. Strategic Management Journal, 30(5), 557-574.
Moller, K., \& Anttila, M. (1987). Marketing capability: A key success factor in small business? Journal of Marketing Management, 3(2), 185-203.

Morgan, N. (2012). Marketing and business performance. Journal of the Academy of Marketing Science, 40(1), 102-119.

Morgan, N., Feng, H., \& Whitler, K. (2018). Marketing capabilities in international marketing. Journal of International Marketing, 26(1), 61-95.

Morgan, N., Katsikeas, C., \& Vorhies, D. (2012). Export marketing strategy implementation, export marketing capabilities, and export venture performance. Journal of the Academy of Marketing Science, 40(2), 271-289.

Morgan, N., Vorhies, D., \& Mason, C. (2009). Market orientation, marketing capabilities, and firm performance. Strategic Management Journal, 30(8), 909-920.

Morgan, N., Zou, S., Vorhies, D., \& Katsikeas, C. (2003). Experiential and informational knowledge, architectural marketing capabilities, and the adaptive performance of export ventures: A cross-national study. Decision Sciences, 34(2), 287-321.

Morschett, D., Schramm-Klein, H., \& Swoboda, B. (2010). Decades of research on market entry modes: What do we really know about external antecedents of entry mode choice? Journal of International Management, 16(1), 60-77.

Müllner, J. (2016). From uncertainty to risk - a risk management framework for market entry. Journal of World Business, 51(5), 800-814.

Musso, F., \& Francioni, B. (2014). International strategy for SMEs: Criteria for foreign markets and entry modes selection. Journal of Small Business and Enterprise Development, 21(2), 301-312.

Nakos, G., Dimitratos, P., \& Elbanna, S. (2019). The mediating role of alliances in the international market orientation-performance relationship of SMEs. International Business Review,

Nath, P., Nachiappan, S., \& Ramanathan, R. (2010). The impact of marketing capability, operations capability and diversification strategy on performance: A resource-based view. Industrial Marketing Management, 39(2), 317-329. 
Papadopoulos, N., \& Martín, O. (2011). International market selection and segmentation: Perspectives and challenges. International Marketing Review, 28(2), 132-149.

Pham, T., Monkhouse, L., \& Barnes, B. (2017). The influence of relational capability and marketing capabilities on the export performance of emerging market firms. International Marketing Review, 34(5), 606-628.

Pinho, J. C., \& Prange, C. (2016). The effect of social networks and dynamic internationalization capabilities on international performance. Journal of World Business, 51(3), 391-403.

Root, F. (1994). Entry strategies for international markets. New York, NY: Lexington books.

Shen, Z., Puig, F., \& Paul, J. (2017). Foreign market entry mode research: $A$ review and research agenda. The International Trade Journal, 31(5), 429456.

Slotegraaf, R., \& Dickson, P. (2004). The paradox of a marketing planning capability. Journal of the Academy of Marketing Science, 32(4), 371-385.

Stal, E., \& Cuervo-Cazurra, A. (2011). The investment development path and FDI from developing countries: The role of pro-market reforms and institutional voids. Latin American Business Review, 12(3), 209-231.

Stoian, C., \& Mohr, A. (2016). Outward foreign direct investment from emerging economies: Escaping home country regulative voids. International Business Review, 25(5), 1124-1135.

Takeuchi, R., Tesluk, P., Yun, S., \& Lepak, D. (2005). An integrative view of international experience. Academy of Management Journal, 48(1), 85-100.

Tallman, S., \& Fladmoe-Lindquist, K. (2002). Internationalization, globalization, and capabilitybased strategy. California Management Review, 45(1), 116-135.

Tan, Q., \& Sousa, C. (2015). Leveraging marketing capabilities into competitive advantage and export performance. International Marketing Review, 32(1), 78-102.
Teece, D., Pisano, G., \& Shuen, A. (1997). Dynamic capabilities and strategic management. Strategic Management Journal, 18(7), 509-533.

Tseng, C., \& Lee, R. (2010). Host environmental uncertainty and equity-based entry mode dilemma: The role of market linking capability. International Business Review, 19(4), 407-18.

Vorhies, D., \& Morgan, N. (2005). Benchmarking marketing capabilities for sustainable competitive advantage. Journal of Marketing, 69(1), 80-94.

Vorhies, D., Morgan, R., \& Autry, C. (2009). Product-market strategy and the marketing capabilities of the firm: Impact on market effectiveness and cash flow performance. Strategic Management Journal, 30(12), 1310-1334.

Wilson, H., \& Daniel, E. (2007). The multi-channel challenge: A dynamic capability approach. Industrial Marketing Management, 36(1), 10-20.

Wu, J. (2013). Marketing capabilities, institutional development, and the performance of emerging market firms: A multinational study. International Journal of Research in Marketing, 30(1), 36-45.

Zaheer, S. (1995). Overcoming the liability of foreignness. Academy of Management Journal, 38(2), 341-363.

Zacharakis, A. (1997). Entrepreneurial entry into foreign markets: A transaction cost perspective. Entrepreneurship Theory \& Practice, 21(3), 23-39.

Zhao, H., Luo, Y., \& Suh, T. (2004). Transaction cost determinants and ownership-based entry mode choice: A meta-analytical review. Journal of International Business Studies, 35(6), 524-544.

Zhao, H., Ma, J., \& Yang, J. (2017). 30 years of research on entry mode and performance relationship: A meta-analytical review. Management International Review, 57(5), 653-682.

Zhao, S., \& Priporas, C-V. (2017). Information technology and marketing performance within international market-entry alliances: A review and an integrated conceptual framework. International Marketing Review, 34(1), 5-28. 


\title{
Sobre os autores:
}

Thiago Chiorino Costa - Universidade Nove de Julho - UNINOVE, São Paulo, (Brasil). E-mail: thiagochiorino@uol.com.br Orcid id: https://orcid.org/0000-0002-0666-1219

Manuel Portugal Ferreira - Universidade Federal de Lavras - UFLA, Minas Gerais, (Brasil). E-mail: manuel.portugal.ferreira@gmail.com Orcid id: http://orcid.org/0000-0002-4642-4605

Julio Araujo Carneiro da Cunha - Universidade Nove de Julho - UNINOVE, São Paulo, (Brasil). E-mail: juliocunha@uni9.pro.br Orcid id: https://orcid.org/0000-0002-1435-055X

Cláudia Frias Pinto - Universidade do Sul de Santa Catarina - UNOESC - Florianópolis, SC (Brasil). E-mail: claudia.frias.pinto@gmail.com Orcid id: https://orcid.org/0000-0002-5250-8848

\section{HOW MARKETING CAPABILITIES INFLUENCE THE CHOICE OF THE ENTRY MODES INTO FOREIGN MARKETS AND HOST COUNTRY SELECTION}

\author{
Thiago Chiorino Costa, Manuel Portugal Ferreira, Julio Araujo Carneiro da Cunha, Cláudia Frias Pinto \\ $\Omega$ Universidade Federal de Lavras - UFLA, Minas Gerais (Brasil) \\ Universidade do Sul de Santa Catarina - UNOESC, Santa Catarina (Brasil) \\ Universidade Nove de Julho - UNINOVE, São Paulo (Brasil)
}

\section{ARTICLE DETAILS}

\section{Article history:}

Received: 08 March 2019

Accepted: 20 November 2019

Available online January: 01 th 2020

Double Blind Review System

\section{Scientific Editor}

Ilan Avrichir

\section{Key words}

Marketing capabilities,

Entry modes,

Foreign market selection

\section{RESUMO}

Goal: In this paper we analyze, conceptually, the influence of marketing capabilities as antecedents of two core decisions for the internationalization of firms: foreign entry mode and host market selection.

Method: We utilize the typology of four types of marketing capabilities specialized, multifunctional, architectural and dynamic - to observe how they may differently induce different choices regarding foreign host markets (location) and foreign entry modes.

Main Results - We propose that higher order marketing capabilities induce internationalization to more developed markets and using entry modes that ensure greater control and commitment of resources and, conversely, that lower order marketing capabilities lead to the choice of similarly or less developed markets using entry modes that favor lower control and less commitment of resources.

Relevance / Originality: Our work explores a possible relationship between companies' marketing capabilities and their influence on the choice of mode of entry into foreign markets and the selection of the destination country.

Theoretical contributions: We thus complement extant research on the role of capabilities in the internationalization of firms, specifically delving into the capabilities that are closer to the market, in contrast to studies on technological knowledge-based competencies or innovation, operational or capabilities based on the ability to arbitrage across markets. 


\title{
CÓMO LAS CAPACIDADES DE MARKETING DETERMINAN LA ELECCIÓN DE MODOS DE ENTRADA EN EL EXTRANJERO Y LA SELECCIÓN DE PAÍSES DE DESTINO
}

\author{
Thiago Chiorino Costa, Manuel Portugal Ferreira, Julio Araujo Carneiro da Cunha, Cláudia Frias Pinto \\ ऽUniversidade Federal de Lavras - UFLA, Minas Gerais (Brasil) \\ Universidade do Sul de Santa Catarina - UNOESC, Santa Catarina (Brasil) \\ Universidade Nove de Julho - UNINOVE, São Paulo (Brasil)
}

\section{DETALLES DEL ARTÍCULO}

\section{Historia del Artículo:}

Recibido: 08 Marzo 2019

Aceptado: 05 Noviembre 2019

Disponible en línea: 01 de enero 2020

\section{Double Blind Review System}

Editor Científico

Ilan Avrichir

\section{Palabras-clave:}

Capacidades de marketing

Modos de entrada

Selección de mercado

\section{RESUMO}

Objetivo: en este documento analizamos conceptualmente la influencia de las capacidades de marketing como antecedentes de dos decisiones fundamentales en la internacionalización de las empresas: la selección del modo de entrada en el extranjero y el país de destino.

Método: Utilizamos la tipología con cuatro tipos de capacidades de marketing: especializadas, multifuncionales, arquitectónicas y dinámicas, para ver cómo pueden, de diferentes maneras, conducir a diferentes opciones de países de destino (ubicación) y modos de entrada.

Resultados clave: Proponemos que las capacidades de marketing de alto nivel induzcan la internacionalización a los países más desarrollados y que utilicen modos de mayor control de entrada y compromiso de recursos, y en contraste, las capacidades de menor nivel que elijan mercados de desarrollo idénticos o inferiores. y modos de entrada con menos control y compromiso de recursos.

Relevancia / Originalidad: nuestro trabajo explora una posible relación entre las capacidades de marketing de las empresas y su influencia en la elección del modo de entrada a los mercados extranjeros y la selección del país de destino.

Contribuições teóricas/metodológicas: Necessário Indicar as principais implicações teóricas e/ou metodológicas que foram alcançadas por meio dos achados do estudo realizado.

Contribuciones teóricas: Complementamos la literatura existente sobre el papel de las capacidades en la internacionalización de las empresas, específicamente al observar las capacidades de marketing más cercanas al mercado, en contraste con la investigación en competencias basadas en tecnología o innovación, habilidades operativas o de habilidades de arbitraje entre mercados.

\section{Para citar este artigo:}

Costa, T., Ferreira, M., Carneiro da Cunha, J., \& Pinto, C. (2020). Como as Capacidades de Marketing Determinam a Escolha dos Modos de Entrada no Estrangeiro e a Seleção dos Países de Destino. Internext, 15(1), 53-70. doi: http://dx.doi.org/10.18568/internext.v15i1.531 